\title{
Exponential Decay of Bound State Wave Functions *
}

\author{
A. J. O'Connor \\ School of Theoretical Physics, Dublin Institute for Advanced Studies, Dublin
}

Received February 1, 1973

\begin{abstract}
The spatial decay properties of the wave functions of multiparticle systems are investigated. The particles interact through pair potentials in the class $R+L_{\varepsilon}^{\infty}$. The bound states lie below the bottom of the continuous spectrum of the system. Exponential decay, in an $L^{2}$ sense, is proven for these wave functions. The result is the best possible one which will cover every potential in this class.
\end{abstract}

\section{Introduction}

In every exactly soluble quantum mechanical two body problem the bound state wave functions fall off exponentially. The rate of exponential decay depends only on the energy of the bound state and the masses of the particles involved. For several particles intuitive arguments on bound states near the bottom of the continuum suggest that for these systems too the wave functions decay exponentially and that the rate of decay depends only on the particle masses and the depth of the bound state below the bottom of the continuum [15]. These $L^{2}$ wave functions are initially just in the domain of the system Hamiltonian. When the potentials can be written as the sum of a bounded function and an $L^{2}$ function with compact support Kato [8] has shown that the wave functions are actually bounded Holder continuous functions. Hunziker [7] and Simon [14] have observed that one way to state a decay result is as an $L^{2}$ domain condition.

For the class of potentials considered by Kato, Ahlrichs [1] has shown that $L^{2}$ conditions imply pointwise decay. This rate of decay is weaker than the $L^{2}$ decay but Simon [25] has recently shown that for a slightly different class of potentials the pointwise decay is precisely the same as the $L^{2}$ decay. We will prove the following theorem.

Theorem. Scalar particles with masses $m_{i}(1 \leqq i \leqq N)$ interacting through local potentials $V_{i j} \in R+L_{\epsilon}^{\infty}$ have a bound state at energy $-E, E>E_{0}$

* Based on a thesis submitted to Princeton University in partial fulfillment of the degree of Doctor of Philosophy. 
where $-E_{0}$ is the bottom of the continuous spectrum for the system Hamiltonian

$$
\hat{H}=\hat{H}_{0}+\sum_{1 \leqq i<j \leqq N} V_{i j} .
$$

$\hat{H}_{0}$ is the free Hamiltonian with the centre of mass motion removed. The wave function $\psi$ for this bound state is in $L^{2}\left(R^{3 N-3}\right)$ and obeys the Schroedinger equation

$$
\hat{H} \psi=-E \psi .
$$

Then $\psi \in D\left[e^{\theta \sqrt{2 M\left(E-E_{0}\right)} r(x)}\right]$ for every $\theta: 0 \leqq \theta<1$ and $x$ measures the positions of the $N$ particles relative to their centre of mass.

$M$ is the total mass of the system and $r(x)$ is defined by $M^{2}(x)$ is the moment of inertia of the $N$ particles about their centre of mass.

We recall that when the interparticle potentials are in $L^{2}+L_{\varepsilon}^{\infty}$, $\hat{H}$ is defined as the operator sum of $\hat{H}_{0}$ and $V=\sum_{i j} V_{i j}$ on their common domain $D\left(\hat{H}_{0}\right)$. When the potentials are in $R+L_{\varepsilon}^{\infty}, \hat{H}$ must be defined by quadratic form techniques. In this case we cannot say that $D(\hat{H})=D\left(\hat{H}_{0}\right)$ $[14$, p. 32].

Previous results have been mostly proven for particular applications. Consequently the methods are fairly direct and generally use a configuration space approach. Hunziker [7] and Combes [3] have proven domain restrictions. The strongest result, [3], is that the bound state wave functions belong to all the spaces. $D_{n}=D\left[|x|^{n}\left(1+\hat{H}_{0}\right)\right]$ for $L^{2}+L_{\epsilon}^{\infty}$ systems. Schnoll [13] uses differential equation methods to show that if the potentials are continuous and bounded below then the wave functions are actually continuous and are bounded by a decreasing exponential. The rate of decay is weaker than ours. Schminke [20] has extended these results to more general situations. More is known about two body systems. Using a configuration space approach Simon [14] proved our theorem for two body Rollnik system. de Alfaro and Regge $[12$, p. 196] have given the best possible result for two body systems with central potentials. They prove that if the central potential is in $L^{\prime} \cap L^{2}\left(R^{3}\right)$ then there is constant $A$, so that $|\psi(x)|<A \frac{e^{-k|x|}}{|x|}$. Ahlrichs [1] has recently proven a pointwise bound for several particle systems of the type considered by Kato [8]. The method used in the paper was suggested by a paper of Slaggie and Wichmann [15]. They used an integral equation approach to obtain exponential decay for a three body system. Their exact result seems to depend on the angular distribution of the three particles and is slightly weaker than the theorem stated here. Nevertheless they were the first to notice the analytic properties of the wave function in momentum space which form the basis for our proofs. 
Section I gives the proof for two particle systems. This contains all the ideas needed for several particle systems. In Section II some properties of the Weinberg-van Winter kernels are established and in Section III the proof for several particle systems is given. An appendix contains certain kinematic definitions.

\section{Section I: Decay for Two Body Systems}

The simplest two body system is when the potential is in $L^{2}$. We can extend this by an approximation argument to $L^{2}+L_{\varepsilon}^{\infty}$ potentials and finally by symmetrization and approximation to $R+L_{\varepsilon}^{\infty}$ potentials. A bound state wave function is an eigen function of $\hat{H}=\hat{H}_{0}+V$ at energy $-E(E>0)$.

Equivalently

$$
\left(\hat{H}_{0}+V\right) \psi=-E \psi \text {. }
$$

$$
\hat{\psi}(p)=\frac{-2 m}{2 m E+p^{2}} \int \hat{V}(p-q) \hat{\psi}(q) d q
$$

$m$ is the reduced mass of the system.

$\hat{\psi}$ the Fourier transform of $\psi$.

A theorem of Paley and Wiener [10] provides the basic tool we need to prove our result.

Theorem 1. A function $f$ in $L^{2}\left(R^{n}\right)$ is in the domain of the multiplication operators $e^{\theta a|x|}$, for all $\theta: 0 \leqq \theta<1$, if and only if its Fourier transform $\hat{f}$ has a representative which can be extended to a function which

(a) is analytic in the complex tube $T_{a}$

$$
T_{a}=\left\{x+i y: x, y \in R^{n},|y|<a\right\} .
$$

(b) has square integrable slices parallel to $R^{n}$, i.e.

$$
\int_{R^{n}}|\hat{f}(x+i y)|^{2} d y<\infty \quad \text { when } \quad|y|<a .
$$

Proof. Paley and Wiener [10] or Titchmarsh [16].

To prove our two body result we construct an analytic continuation of $\hat{\psi}$ to a tube $T(E)$ in $C^{3}$. Quite formally if an extension existed it would satisfy the continuation of (2).

$$
\hat{\psi}(p+i r)=\frac{-2 m}{2 m E+(p+i r)^{2}} \int \hat{V}(p-q) \hat{\psi}(q+i r) d q .
$$

Starting from this equation we construct an $L^{2}\left(R^{3}\right)$ valued extension of $\hat{\psi}$ to the tube $T(E)$ and then piece together these $L^{2}$ functions to give a function which is analytic in the whole tube. $T(E)$ is just $T_{k}$ with $k^{2}=2 m E$. 
Definition 1. $K(z, E)$ is the integral operator on $L^{2}\left(R^{3}\right)$ whose kernel is

$$
\frac{-2 m}{(p+z)^{2}+2 m E} \hat{V}(p-q) \quad\left(V \in L^{2}, z \in T(E)\right) .
$$

Definition 2. $U(t)$ is the translation group on $L^{2}\left(R^{3}\right)$, i.e.

$$
[U(t) f](p)=f(p+t) .
$$

Lemma 1. (a) $K(z, E)$ is an analytic family of Hilbert Schmidt operators in the tube $T(E)$.

(b) The operators $K(z, E)$ have the translation property

$$
U(t) K(z, E) U(t)^{-1}=K(z+t, E) .
$$

(c) If $-E \in \sigma_{p p}(\hat{H}), 1 \in \sigma[K(z, E)]$ for every $z \in T(E)$.

(d) If $P(z)$ is the projection onto this eigenspace then $P(z)$ is an analytic family of finite rank projections and

$$
U(t) P(z) U(t)^{-1}=P(z+t) .
$$

Proof. (a) and (b) are obvious. Schroedinger's equation and (b) show that $1 \in \sigma[K(t, E)]$ for all $t \in R^{3}$. The analytic Fredholm theorem (Reed and Simon [11], Hunziker [6]) shows that (c) is true. (d) follows from the Cauchy representation for $P(z)$

(when $\varepsilon$ is small).

$$
2 \pi i P(z)=\int_{|\omega-1|=\varepsilon}[\omega-K(z, E)]^{-1} d \omega
$$

$P(z)$ is the projection onto the eigenspace of $K(z, E)$ corresponding to the eigenvalue 1 . This is so if $[\lambda-K(z, E)]^{-1}$ has a simple pole at $\lambda=1$. A simple calculation checks this at $z=0$ and the translation property and analyticity in $z$ show that it is also true for each $z$ in $T(E)$.

The next theorem enables us to choose an analytic family of eigenvectors from the different eigenspaces.

Theorem 2. If $P(z)$ is an analytic family of finite rank projections on $H$ in a tubular domain $D=A+i R^{n}$ ( $A$ is open in $R^{n}$ ) and

$$
U(t) P(z) U(t)^{-1}=P(z+t)
$$

for a unitary group $U(t)\left(t \in R^{n}\right)$ then when $\psi \in R$ an $P(0)$, the vector valued function $U(t) \psi$ has an analytic vector valued extension to the tube $D$.

Proof. If the self adjoint operator $A$ is the generator of the unitary group $U(t)$ on $H$, the set $\mathscr{E}$ of entire vectors for $A$ is dense in $H$ [19]. So the linear span of the set $P(0) \mathscr{E}$ is just $\operatorname{Ran} P(0)$. Thus we can choose 
entire vectors $e_{1} \ldots e_{n}$ so that $\left\{P(0) e_{i}: i=1 \ldots n\right\}$ is an orthonormal basis for $\operatorname{Ran} P(0)$ and so any vector $\psi \in \operatorname{Ran} P(0)$ can be written as

If $t \in R^{n}$

$$
\psi=\sum_{i=1}^{n}\left(e_{i}, \psi\right) P(0) e_{i} .
$$

$$
\psi(t)=U(t) \psi=\sum_{i=1}^{n}\left(e_{i}, \psi\right) P(t) U(t) e_{i}
$$

has an analytic extension to all of $D$ given by

$$
\psi(z)=\sum_{i=1}^{n}\left(e_{i}, \psi\right) P(z) e^{i z A} e_{i} .
$$

This is well defined since $e_{i} \in \mathscr{E}$. Notice that $\psi(z)$ is in the range of $P(z)$ and that

$$
\begin{aligned}
U(t) \psi(z) & =\sum_{i=1}^{n}\left(e_{i}, \psi\right) U(t) P(z) e^{i z A} e_{i} \\
& =\sum_{i=1}^{n}\left(e_{i}, \psi\right) \cdot P(z+t) U(t) e^{i z A} e_{i} \\
& =\psi(z+t) .
\end{aligned}
$$

So $\psi(z)$ cannot vanish at a single point without vanishing on a whole hyperplane and so vanishing identically in $D$. Hence $\psi(z)$ never vanishes inside $D$.

Remark. The finite dimensionality of $\operatorname{Ran} P(0)$ enters when we observe that any dense subspace of $\operatorname{Ran} P(0)$ is of necessity all of $\operatorname{Ran} P(0)$. The example $P(z)=I$ shows that finite dimensionality is critical.

Lemma 2. If $\hat{H} \psi=-E \psi$, where $\hat{H}_{0}=\hat{H}_{0}+V$ with $V \in L^{2}\left(R^{3}\right)$, then the Fourier transform of $\psi, \hat{\psi}$, has an $L^{2}$ valued extension $\hat{\psi}_{z}$ to the tube $T(E)$ such that $\hat{\psi}_{0}=\hat{\psi}$ and $\hat{\psi}_{z+t}=U(t) \hat{\psi}_{z}$ for $z \in T(E)$ and $t \in R^{3}$.

Proof. Lemma 1 and Theorem 2. $\hat{\psi}_{z}$ satisfies the integral equation

$$
\hat{\psi}_{z}(p)=\frac{-2 m}{2 m E+(p+z)^{2}} \int \hat{V}(p-q) \hat{\psi}_{z}(q) d q .
$$

Lemma 3. If $\hat{\psi}_{z}$ is the family of $L^{2}$ functions defined by Lemma 2 then (a) We can choose a representative for each $\hat{\psi}_{z}$ which is in $C_{0}\left(R^{3}\right)$. In this way $\hat{\psi}_{z}(p)$ has a unique interpretation.

(b) For each fixed $p, \hat{\psi}_{z}(p)$ is an analytic function of $z$ in $T(E)$.

(c) $\hat{\psi}_{z}(p+t)=\hat{\psi}_{z+t}(p)$.

Proof. (a) $\hat{\psi}_{z}$ is the product of a $C_{0}$ function and the convolution of two $L^{2}$ functions. (b) $\hat{\psi}_{z}$ can be written as a power series in $z$ whose coefficients are $L^{2}$ functions. (c) $\hat{\psi}_{z+t}(p)=\left[U(t) \hat{\psi}_{z}\right](p)=\hat{\psi}_{z}(p+t)$. 
Definition 3. We can sensibly define an analytic function in $T(E)$ by

$$
\hat{\Psi}(z)=\hat{\psi}_{z}(0) \text {. }
$$

Lemma 4. $\hat{\Psi}(p)$ is just the Fourier transform of $\psi$ and for each $q \in R^{3}$, with $|q|^{2}<2 m E$

$$
\hat{\Psi}(\cdot+i q) \in L^{2}\left(R^{3}\right) .
$$

Proof. $\hat{\Psi}(p+i q)=\hat{\psi}_{i q}(p)$ and $\hat{\psi}_{i q} \in L^{2}$

$$
\hat{\Psi}(p)=\left[U(p) \hat{\psi}_{0}\right](0)=[U(p) \hat{\psi}](0)=\hat{\psi}(p) .
$$

Theorem 3. If $V \in L^{2}\left(R^{3}\right)$ and $\psi$ is a bound state of $\hat{H}=\hat{H}_{0}+V$ at energy $-E$ then

$$
\psi \in D\left[e^{\theta \sqrt{2 m E}|x|}\right]
$$

for each $\theta: 0 \leqq \theta<1 . m$ is the reduced mass of the system.

Proof. Lemma 4 and Theorem 1.

We cannot directly carry over all the steps in the $L^{2}$ proof to $L^{2}+L_{\varepsilon}^{\infty}$ potentials. Lemma 3 cannot be proven directly. The approximation argument we use is also the basis for the Rollnik proofs.

Definition 4. If $V \in L^{2}+L_{\varepsilon}^{\infty}$ we can choose potentials $V_{n} \in L^{2}$ so that $\left\|V-V_{n}\right\|_{\infty}<\frac{1}{n}$ and we can define operators

$$
\begin{aligned}
K(z, E) & =G_{0}(z, E) V, \\
K_{n}(z, E) & =G_{0}(z, E) V_{n} .
\end{aligned}
$$

In the momentum representation $G_{0}(z, E)$ is multiplication by

$$
\frac{-2 m}{2 m E+(p+z)^{2}} \text {. }
$$

Lemma 5. (a) $K_{n}(z, E)$ is a sequence of HS operators which converge in norm to the compact operator $K(z, E)$. Convergence is uniform on compact subsets of $T(E)$. The operators $K(z, E)$ form an analytic family of compact operators in $T(E)$.

(b) $U(t) K(z, E) U(t)^{-1}=K(z+t, E)$ for $z \in T(E)$ and $t \in R^{3}$.

(c) If $-E \in \sigma_{p p}(\hat{H}), 1 \in \sigma[K(z, E)]$ for each $z \in T(E)$.

Proof. Very similar to Lemma 1.

Definition 5. If $\varepsilon$ is so small that

then

$$
(1-\varepsilon, 1+\varepsilon) \cap \operatorname{spec}[K(0, E)]=\{1\}
$$

$$
(1-\varepsilon, 1+\varepsilon) \cap \operatorname{spec}[K(z, E)]=\{1\}
$$


for all $z$ in $T(E)$ and we can define $P(z)$ as the projections onto the eigenspaces of $K(z, E)$ corresponding to the eigenvalue 1 and if $n$ is large enough

$$
P_{n}(z)=\frac{1}{2 \pi i} \int_{|\omega-1|=\varepsilon}\left[\omega-K_{n}(z, E)\right]^{-1} d \omega
$$

is also well defined for each $z$ in $T(E)$.

Lemma 6. (a) $P(z)$ and $P_{n}(z)$ are analytic families of projections in the tube $T(E)$. They each have the translation property (4).

(b) $P_{n}(z)$ converges in norm to $P(z)$, uniformly on compact subsets of $T(E)$.

Proof. Straightforward.

Lemma 7. If $V \in L^{2}+L_{\varepsilon}^{\infty}$ and $\hat{H}=\hat{H}_{0}+V$ has a bound state $\psi$ at energy $-E(E>0)$ then

(a) Its Fourier transform $\hat{\psi}$ has an $L^{2}$ valued analytic extension $\hat{\psi}_{z}$ which agrees with $U(t) \psi$ when $z \in R^{3}$.

(b) If $\hat{\psi}_{n, z}=P_{n}(z) \hat{\psi}_{z}$, then $\hat{\psi}_{n, z}$ is also an $L^{2}$ valued analytic function in $T(E)$. For each $n$, the functions $\hat{\psi}_{n, z}$ are slices of analytic functions $\hat{\Psi}_{n}(z)$ which satisfy the Paley-Wiener condition.

(c) The slices $\hat{\psi}_{n, z}$ converge in $L^{2}$ to the $L^{2}$ functions $\hat{\psi}_{z}$. The functions $\hat{\Psi}_{n}(z)$ converge at each point of $T(E)$ to an analytic function $\hat{\Psi}(z)$ on $T(E)$.

Proof. (a) Theorem 2 and Lemma 6.

$$
\begin{aligned}
\hat{\psi}_{n, t} & =P_{n}(t) \hat{\psi}_{t}=P_{n}(t) U(t) \hat{\psi}_{0} \\
& =U(t) P_{n}(0) \hat{\psi}_{0}=U(t) \hat{\psi}_{n, 0} .
\end{aligned}
$$

Theorem 2 guarantees that $P_{n}(z) \hat{\psi}_{z}$ is a continuation of $U(t) \hat{\psi}_{n, 0}$ to the tube $T(E) . \hat{\psi}_{n, 0}$ is a linear combination of the eigenfunctions of $K_{n}(0)$ whose eigenvalues are between $1-\varepsilon$ and $1+\varepsilon$. Suppose these are $\theta_{1}^{(n)} \ldots \theta_{p}^{(n)}$ and that they correspond to eigenvalues $\lambda_{1} \ldots \lambda_{p}$. Each $\theta_{j}^{(n)}$ has an $L^{2}$ valued analytic extension $\Theta_{j, z}^{(n)}$ to $T(E)$.

$$
\Theta_{j, z}^{(n)}=\lambda_{j} K_{n}(z, E) \Theta_{j, z}^{(n)} .
$$

For fixed $z$, this is a continuous function of $p$ and for fixed $p$ an analytic function of $z$. So the $L^{2}$ valued analytic functions $\hat{\psi}_{n, z}$ are just slices of an analytic function $\hat{\Psi}_{n}(z)$. The functions $\hat{\psi}_{n, z}$ converge in $L^{2}$ to $\hat{\psi}_{z}$ and the convergence is uniform on compact subsets. We see that $\hat{\Psi}_{n}(z)$ is a Cauchy sequence at each point of $T(E)$ from the Cauchy integral formula which gives

$$
\pi \delta^{2}\left|\hat{\Psi}_{n}(z)-\hat{\Psi}_{m}(z)\right|^{2} \leqq \int_{-\delta}^{\delta} d y \int_{R^{\prime}} d x\left|\hat{\Psi}_{n}(z+x+i y)-\hat{\Psi}_{m}(z+x+i y)\right|^{2} .
$$


Hence we can define an analytic function by

$$
\hat{\Psi}(z)=\lim _{n \rightarrow \infty} \hat{\Psi}_{n}(z) .
$$

Theorem 4. If $V \in L^{2}+L_{\varepsilon}^{\infty}$ and $\psi$ is a bound state of $\hat{H}$ at energy $-E(E>0)$ then for each $\theta: 0 \leqq \theta<1$

$$
\psi \in D\left[e^{\theta \sqrt{2 m E}|x|}\right] .
$$

Proof. We need only check that $\hat{\Psi}$ obeys the conditions of the Paley-Wiener theorem. $\hat{\Psi}(p)$ is just the Fourier transform of $\psi$ and $\hat{\Psi}(.+i y)=\hat{\psi}_{i y}(.) \in L^{2}\left(R^{3}\right)$ for each $y:|y|^{2}<2 m E$. The extension is consistent because

$$
\lim _{n \rightarrow \infty} \hat{\psi}_{n, z}(x)=\lim _{n \rightarrow \infty} \hat{\psi}_{n}(x+z)=\hat{\Psi}(x+z) .
$$

Theorem 4 is also true for two body Rollnik systems. When $V \in R$ or $R+L_{\varepsilon}^{\infty}, \hat{H}$ is defined by a quadratic form method [14]. While we cannot say that $D\left(\hat{H}_{0}\right)=D(\hat{H})$ we do know that

$$
D(\hat{H}) \subset Q\left(\hat{H}_{0}\right)=D\left(\hat{H}_{0}^{1 / 2}\right) .
$$

If $\psi$ is a bound state of $\hat{H}$ with energy $-E$ then

and

$$
\phi=\left(E+\hat{H}_{0}\right)^{\frac{1}{2}} \psi \in L^{2}\left(R^{3}\right)
$$

$$
\phi=G_{0}(-E)^{\frac{1}{2}} V G_{0}(-E)^{\frac{1}{2}} \phi .
$$

Conversely any solution of (9) produces an eigenfunction of $\hat{H}$ at energy $-E$. Both claims follow from

$$
G(z)=G_{0}^{\frac{1}{2}}(z)\left[1-G_{0}^{\frac{1}{0}}(z) V G_{0}^{\frac{1}{2}}(z)\right]^{-1} G_{0}^{\frac{1}{2}}(z)
$$

$[14, p .45]$.

To prove exponential fall off for the wave function it is enough to prove that $\hat{\phi}$ has an analytic extension to $T(E)$ which obeys the PaleyWiener conditions. This proof is very similar to that for $L^{2}+L_{\varepsilon}^{\infty}$ potentials.

Definition 6. $K^{s}(z, E)=G_{0}(z,-E)^{\frac{1}{2}} V G_{0}(z,-E)^{\frac{1}{2}}$ and if $V_{n}$ is a sequence of potentials in $R \cap L^{2}$ converging to $V$ in $R$ then

$$
K_{n}^{s}(z, E)=G_{0}(z,-E)^{\frac{1}{2}} V_{n} G_{0}(z,-E)^{\frac{1}{2}} .
$$

Lemma 8. (a) $K_{n}^{s}(z, E)$ is an analytic family of HS operators on $T(E)$. For fixed $z$ these operators converge to $K^{s}(z, E)$. The convergence is uniform on compact subsets of $T(E)$ and so $K^{s}(z, E)$ is an analytic family of compact operators.

(b) Both $K^{s}(z, E)$ and $K_{n}^{s}(z, E)$ have the translation property (4).

(c) If $\hat{H} \psi=-E \psi$, then the Fourier transform $\hat{\psi}$ of $\psi$ has an $L^{2}$ valued analytic extension $\hat{\psi}_{z}$ to $T(E)$. 
Proof. (a) and (b) are obvious. (c) follows if we show that $\hat{\phi}$ has an extension $\hat{\phi}_{z}$ to $T(E)$ and if we define

$$
\hat{\psi}_{z}(p)=\left[2 m E+(p+z)^{2}\right]^{-\frac{1}{2}} \hat{\phi}_{z}(p) .
$$

(a) and (b) combined with $\hat{\phi}=K^{s}(0, E) \hat{\phi}$ complete the proof.

Theorem 5. If $V \in R+L_{\varepsilon}^{\infty}$ and $\hat{H} \psi=-E \psi$ then

$$
\psi \in D\left[e^{\theta \sqrt{2 m E}|x|}\right]
$$

for every $\theta: 0 \leqq \theta<1$.

Proof. When $V \in R$ an approximation argument using the families $K_{n}^{s}(z, E)$ shows that the $L^{2}$ valued analytic family $\hat{\phi}_{z}$ is just the family of slices of an analytic function $\hat{\Phi}(z)$ in $T(E)$. The slices of $\hat{\Phi}(z)$ parallel to $R^{3}$ are square integrable. This completes the proof for $V \in R$. When $V \in R+L_{\varepsilon}^{\infty}$ an approximation argument using Rollnik potentials will work.

\section{Section II. Continuation of the Weinberg-van Winter Kernel into Tubes}

In the two body system, three properties of $K(0, E)$ were essential in proving the $L^{2}$ result.

(A) $K(0, E)=G_{0}(-E) V$ was a compact operator on $L^{2}\left(R^{3}\right)$.

(B) $K(0, E)$ mapped $L^{2}\left(R^{3}\right)$ into $C_{0}\left(R^{3}\right)$.

(C) $K(0, E)$ has an analytic continuation $K(Z, E)$ to a family of compact operators in a tubular domain in $C^{3}$.

In a several particle system $K(0, E)$ becomes the Weinberg-van Winter kernel $I(-E)$. First we will review the Weinberg-van Winter equation and then we will prove analogues of (A), (B) and (C) for the kernel $I(-E)$. Some definitions are useful.

Definition 7. (a) If $D$ is a decomposition of $\{1 \ldots N\}$ into clusters $\left\{C_{1} \ldots C_{k}\right\}$ then $\hat{H}_{D}=\hat{H}_{0}+V_{D}$ is the cluster Hamiltonian.

$$
V_{D}=\sum_{j=1}^{k} \sum_{a, b \in C_{j}} V_{a b}
$$

(b) A decomposition $D^{\prime}$ is finer than $D$ if it contains more clusters and each cluster in $D^{\prime}$ is contained in one of the clusters of $D$. We write $D^{\prime} \supset D$.

(c) If $D$ and $D^{\prime}$ are cluster decompositions then $D^{\prime} \supset D$ if $D^{\prime}$ is a strictly finer decomposition of $D$ and if $D$ is obtained from $D^{\prime}$ by joining just two of the clusters in $D^{\prime}$.

(d) A string $S$ of cluster decompositions is a sequence $\left\{D_{j} \ldots D_{k}\right\}$ of decompositions in which $D_{j} \supset_{1} D_{j+1} \supset_{1} \cdots \supset_{1} D_{k} D_{l}$ contains $l$ clusters so that $k \leqq j$. 
(e) If $D^{\prime} \supset D$ then $V_{D^{\prime} D}=V_{D}-V_{D^{\prime}}$.

(f) The string $S$ is disconnected if $D_{k} \neq\{1 \ldots N\}$. It is connected if $k=1$ and $D=\{1 \ldots N\}$. More generally a string $\left\{D_{N} \ldots D_{l}\right\}$ is $D$ connected if $D \supset D_{l}$ and $D$ disconnected if $D_{l} \supset D$.

Definition 8. When the interparticle potentials $V_{i j} \in L^{2}+L_{\varepsilon}^{\infty}$ the disconnected part of the Green's function $D(z)$ and the Weinberg kernel $I(z)$ are

$$
D(z)=\sum_{\substack{S=\left\{D_{N} \ldots D_{k}\right\} \\ k \neq 1}} G_{D_{N}}(z) V_{D_{N} D_{N-1}} G_{D_{N-1}}(z) \ldots G_{D_{2}}(z) V_{D_{2} D_{1}} .
$$

The sum is over all disconnected strings $S$.

$$
I(z)=\sum_{S=\left\{D_{N} \ldots D_{1}\right\}} G_{D_{N}}(z) V_{D_{N} D_{N-1}} \ldots G_{D_{2}}(z) V_{D_{2} D_{1}} .
$$

This sum is over all connected strings $S$.

Theorem 6. When $z \notin \sigma(\hat{H})$ and all the interparticle potentials are in $L^{2}+L_{\varepsilon}^{\infty}$ the Weinberg-van Winter equation holds, i.e.

$$
G(z)=D(z)+I(z) G(z) .
$$

$D(z)$ is an analytic family of bounded operators when $z \notin \sigma_{c}(\hat{H})$.

$I(z)$ is an analytic family of compact operators when $z \notin \sigma_{c}(\hat{H})$.

Proof. Weinberg [18], van Winter [17].

Corollary. If $-E$ is an isolated eigenvalue of $\hat{H}$ and if $\hat{H} \psi=-E \psi$ then

$$
\psi=I(-E) \psi .
$$

Note that the converse is not true. There might be eigenfunctions of $I(-E)$ which are not eigenfunctions of $\hat{H}$. These are the "spurious zeros" of $I$. ([9], [4]).

When the potentials are in $R+L_{\varepsilon}^{\infty}$ we need the factorized Weinbergvan Winter equation.

Definition 9. The reduced Green's functions $R$ and $R_{D}$ are

$$
\begin{aligned}
R(z) & =G_{0}(z)^{-\frac{1}{2}} G(z) G_{0}(z)^{-\frac{1}{2}}, \\
R_{D}(z) & =G_{0}(z)^{-\frac{1}{2}} G_{D}(z) G_{0}(z)^{-\frac{1}{2}} .
\end{aligned}
$$

The symmetrized disconnected part of the Green's function is

$$
D_{S}(z)=\sum_{\substack{S=\left\{D_{N} \ldots D_{k}\right\} \\ k>1}} R_{D_{N}}(z)\left(G_{0}^{\frac{1}{2}} V_{D_{N} D_{N}-1} G_{0}^{\frac{1}{2}}\right) \ldots R_{D_{k}}(z) .
$$

The symmetrized connected part is

$$
I_{S}(z)=\sum_{S=\left\{D_{N} \ldots D_{1}\right\}}\left(G_{0}^{\frac{1}{2}} V_{D_{N} D_{N-1}} G_{0}^{\frac{1}{2}}\right) R_{D_{N-1}} \ldots R_{D_{2}}\left(G_{0}^{\frac{1}{2}} V_{D_{2} D_{1}} G_{0}^{\frac{1}{2}}\right) .
$$


Theorem 7. When all the interparticle potentials are in $R+L_{\varepsilon}^{\infty}$ and $z \notin \sigma(\hat{H})$ the symmetrized Weinberg-van Winter equation is

$$
R(z)=D_{S}(z)+I_{S}(z) R(z) .
$$

$D_{s}(z)$ and $I_{S}(z)$ are respectively analytic families of bounded and compact operators when

Proof. Simon [14, p. 185].

Corollary. A necessary but not sufficient condition that $\psi$ be an eigenfunction of $\hat{H}$ corresponding to an isolated eigenvalue $-E$ is

where

$$
\phi=I_{S}(-E) \phi
$$

$$
\phi=G_{0}(-E)^{-\frac{1}{2}} \psi .
$$

If we are to carry over the two body proof to several particle systems we have to show that $I(-E)$ and $I_{s}(-E)$ have properties $(\mathrm{A}),(\mathrm{B})$ and $(\mathrm{C})$. (A) is already proven for us so only (B) and (C) must be checked. $I(-E)$ is built from all barely connected diagrams. Each such diagram can be broken into a diagram which just fails to be disconnected followed by an interaction which connects everything together. The barely disconnected diagram represents an integral operator which leaves one of the $N-1$ momenta unchanged but which acts on functions of the remaining $N-2$ momenta as a compact operator. This suggests that we use an induction argument to prove (B) and (C). We must define some more kernels. $C_{D}$ is the sum of all the diagrams which are $D$ connected and $I_{D}$ the sum of all diagrams which are barely $D$ connected.

Definition 10. When the potentials $V_{i j} \in L^{2}+L_{\varepsilon}^{\infty}$ and $z \notin \sigma_{c}\left(\hat{H}_{D}\right)$ the $D$ connected kernel is just

$$
C_{D}(z)=\sum_{\substack{S=\left\{D_{N} \ldots D_{k}\right\} \\ D_{k}=D}} G_{D_{N}} V_{D_{N} D_{N-1}} G_{D_{N-1}} \ldots V_{D_{k+1} D_{k}} G_{D_{k}} .
$$

The $D$ connected Weinberg-van Winter kernel is

$$
I_{D}(z)=\sum_{\substack{S=\left\{D_{N} \ldots D_{k}\right\} \\ D_{k}=D}} G_{D_{N}} V_{D_{N} D_{N-1}} G_{D_{N-1}} \ldots G_{D_{k+1}} V_{D_{k+1} D_{k}} .
$$

Lemma 9. (a) $C_{D}(z)$ and $I_{D}(z)$ are analytic families of bounded operators when $z \notin \sigma_{c}\left(\hat{H}_{D}\right)$,

(b) When $z \notin \sigma_{c}\left(\hat{H}_{D}\right)$,

$$
\begin{aligned}
G_{D}(z) & =\sum_{D^{\prime} \nmid D} C_{D^{\prime}}(z)+I_{D}(z) G_{D}(z), \\
C_{D}(z) & =I_{D}(z) G_{D}(z), \\
I_{D}(z) & =\sum_{D^{\prime} \supset D} I_{D^{\prime}}(z) G_{D^{\prime}}(z) V_{D^{\prime} D} .
\end{aligned}
$$


Proof. (a) It is enough to show that when $A \supset{ }_{1} B, V_{A B} G_{B}(z)$ is a bounded operator. Hunziker's theorem [6] shows that the total set from which $z$ is excluded is just $\sigma_{c}\left(\hat{H}_{D}\right)$.

(b) When $\operatorname{Re} z$ is very negative the perturbation series expansions in terms of diagrams converge. Analytic continuation completes the proof.

The analogue of Lemma 3 would be that the kernel $I(z)$ maps $L^{2}\left(R^{3^{N}-3}\right)$ into $C_{0}\left(R^{3 N-3}\right)$. We will prove a more general result for this new class of kernels $I_{D}(z)$. These really act only on the internal momenta of the clusters in $D$. A simple example of this is the three body system and the decomposition $D=\{(12),(3)\}, I_{D}(z)=G_{0} V_{12}\left(V_{12} \in L^{2}\right)$. We can choose as coordinates the Jacobi scheme, $(p, q) ; p$ is the relative momentum of 1 and $2, q$ is the relative momentum of 3 and the centre of mass of 1 and 2. Ignoring mass factors $I_{D}$ has the kernel

$$
I_{D}(p q, p r)=\left(z-p^{2}-q^{2}\right)^{-1} \hat{V}_{12}(q-r) .
$$

When $p$ is fixed, $I_{D}$ is a $H S$ kernel in $q$ and $r$. More precisely we can examine its action on the functions $f(p, r)=g(p) h(r)$. Then

$$
\begin{aligned}
{\left[I_{D}(z) g \otimes h\right](p, r) } & =\left[\int\left(z-p^{2}-r^{2}\right)^{-1} \hat{V}_{12}(q-r) h(r) d r\right] \cdot g(p) \\
& =\left[\tilde{I}_{D}\left(z-p^{2}\right) h\right](q) \cdot g(p) .
\end{aligned}
$$

$\tilde{I}_{D}$ is just the two body kernel $G_{0} \hat{V}_{12}$. It maps $L^{2}\left(R^{3}\right)$ into $C_{0}\left(R^{3}\right)$. We generalize this simple example. Jacobi coordinates are described in the appendix.

Definition 11. If $D$ is any cluster decomposition of $\{1 \ldots N\}$ then we can choose a set of Jacobi coordinates $p_{D}$ and relative momenta $\tilde{p}_{D}$ for the clusters in $D$ which together will form a complete set of momenta describing the $N$ particles in their centre of mass frame.

$$
L^{2}(D), \quad C_{0}(D), \quad L^{2}(\tilde{D}) \quad \text { and } \quad C_{0}(\tilde{D})
$$

are the spaces and of $L^{2}$ and $C_{0}$ functions in the variables $p_{D}$ and $\tilde{p}_{0}$. It is clear that $L^{2}(D) \otimes L^{2}(\tilde{D})=L^{2}\left(R^{3 N-3}\right)$.

In the following theorems we ignore mass factors. These will be taken into account with a little care later on.

Theorem 8. All the interparticle potentials are in $L^{2}\left(R^{3}\right)$. Each operator $I_{D}(z)$ when acting on functions in $L^{2}\left(R^{3 N-3}\right)$ of the form $f \otimes g$, with $f \in L^{2}(D)$ and $g \in L^{2}(\tilde{D})$ can be written in the form

$$
\left[I_{D}(z) f \otimes g\right]\left(p_{D}, \tilde{p}_{D}\right)=\left[\tilde{I}_{D}\left(z-\tilde{p}_{D}^{2}\right) f\right]\left(p_{D}\right) \cdot g\left(\tilde{p}_{D}\right) .
$$

$\tilde{I}_{D}(z)$ is an analytic family of $H S$ operators acting on $L^{2}(D)$ when $z \notin \sigma_{c}\left(\hat{H}_{D}\right)$. 
Proof. We argue inductively on (26). Each item in (26) has a very simple form in the appropriate Jacobi frame. If $D^{\prime} \supset D$ we can choose a Jacobi frame $p_{D}$ which contains the relative momentum, $p$, of the two clusters in $D^{\prime}$ which are joined together to form a single cluster in $D$, i.e. $p_{D}=\left(p_{D^{\prime}}, p\right)$.

If

then

$$
f\left(p_{D}, \tilde{p}_{D}\right)=F\left(p_{D^{\prime}}\right) \cdot G(p) \cdot H\left(\tilde{p}_{D}\right)
$$

$$
\begin{aligned}
{\left[I_{D^{\prime}} G_{D^{\prime}}(z)\right.} & \left.V_{D^{\prime} D}\right][F \otimes G \otimes H]\left(p_{D^{\prime}}, p, \tilde{p}_{D}\right) \\
& =\left[\tilde{I}_{D^{\prime}} g_{D^{\prime}}\left(z-\tilde{p}_{D}^{2}-p^{2}\right) F\right]\left(p_{D^{\prime}}\right) \cdot\left[V_{D^{\prime} D} G\right](p) \cdot H\left(\tilde{p}_{D}\right) \\
& =\left[J_{D^{\prime} D}\left(z-\tilde{p}_{D}^{2}\right) F \otimes G\right]\left(p_{D^{\prime}}, p\right) \cdot H\left(\tilde{p}_{D}\right)
\end{aligned}
$$

Here

$$
g_{D^{\prime}}(z)=\left(z-\hat{h}_{D^{\prime}}\right)^{-1}
$$

$\hat{h}_{D^{\prime}}=\hat{H}_{0, D^{\prime}}+V_{D^{\prime}}$ is the Hamiltonian describing the internal structure of the clusters in $D^{\prime}$. It is a self adjoint operator on $L^{2}\left(D^{\prime}\right)$ and $\sigma\left(\hat{h}_{D^{\prime}}\right)=\sigma\left(\hat{H}_{D^{\prime}}\right)$.

If all the potentials are in $L^{2}\left(R^{3}\right)$ it is not hard to check that $J_{D^{\prime} D}(z)$ extends from vectors of the type $F \otimes G$ to a $H S$ operator on all of $L^{2}(D)$.

Explicitly if $\left\{a_{i}\right\}$ and $\left\{b_{j}\right\}$ are bases for $L^{2}(D)$ and $L^{2}\left(D D^{\prime}\right)=L^{2}\left(R^{3}\right)$,

$$
\begin{aligned}
\sum_{i, j=1}^{\infty}\left\|J_{D^{\prime} D}(z) a_{i} \otimes b_{j}\right\|^{2} & \\
& =\sum_{i, j=1}^{\infty}\left\|\left(\tilde{I}_{D^{\prime}} g_{D^{\prime}}\left(z-p^{2}\right) a_{i}\right)\left(p_{D^{\prime}}\right) \cdot\left(V_{D^{\prime} D} b_{j}\right)(p)\right\|^{2} \\
& \leqq \sum_{j=1}^{\infty}\|\| \tilde{I}_{D^{\prime}} g_{D^{\prime}}\left(z-p^{2}\right)\left\|_{H S}\left(V_{D^{\prime} D} b_{j}\right)(p)\right\|^{2} \\
& \leqq \sup _{p}\left\{\left\|\tilde{I}_{D^{\prime}}\left(z-p^{2}\right)\right\|_{H S}^{2}\left\|\left(z-E_{D^{\prime}}-p^{2}\right)^{-1} V_{D^{\prime} D}\right\|_{H S}^{2}\right\} \\
& <\infty .
\end{aligned}
$$

$-E_{D^{\prime}}=\inf \sigma\left(\hat{h}_{D^{\prime}}\right)$ and we assume that for every $D^{\prime} \underset{\ddagger}{D}$

$$
\sup _{p}\left\|I_{D^{\prime}}\left(z-p^{2}\right)\right\|_{H S}<\infty \text {. }
$$

The decomposition $D=\{(12),(3) \ldots(N)\}$ is the first step in the induction argument. This is just the two body situation. $\tilde{I}_{D}$ is the sum of these $H S$ operators $J_{D^{\prime} D}$ and so the theorem is true.

Theorem 9. If all the interparticle potentials $V_{i j} \in L^{2}\left(R^{3}\right)$, then $\tilde{I}_{D}(z)$ maps $L^{2}(D)$ into $C_{0}(D)$. 
Proof. Again by induction. The hypothesis is that for each $D^{\prime} \supset D$, $\tilde{I}_{D}(z)$ maps $L^{2}\left(D^{\prime}\right)$ into $C_{0}\left(D^{\prime}\right)$ and that

$$
\left\|\tilde{I}_{D^{\prime}}(z) f\right\|_{C_{0}} \leqq m_{D^{\prime}}(z)\|f\|_{L^{2}}
$$

where $m_{D^{\prime}}$ is finite away from $\sigma_{c}\left(\hat{h}_{D^{\prime}}\right)$ and $m_{D^{\prime}}(z) \rightarrow 0$ as $\operatorname{Re} z \rightarrow-\infty$. The contribution to $\tilde{I}_{D}(z) f$ from the decomposition $D^{\prime}$ is just

$$
\left[\tilde{I}_{D^{\prime}} g_{D^{\prime}}\left(z-p^{2}\right) V_{D^{\prime} D}^{p} f\right]\left(p_{D^{\prime}}\right)=\left[J_{D^{\prime} D}(z) f\right]\left(p_{D^{\prime}}\right)
$$

$V^{p}: L^{2}(D) \rightarrow L^{2}\left(D^{\prime}\right)$ is a family of maps depending continuously on $p$.

$$
\left[V^{b} f\right]\left(p_{D^{\prime}}\right)=\int f\left(p_{D^{\prime}}, q\right) \hat{V}(p-q) d q .
$$

So $J_{D^{\prime} D}$ maps the functions $F \otimes G$, where $F \in L^{2}\left(D^{\prime}\right)$ and $G \in L^{2}\left(D D^{\prime}\right)$ into $C_{0}(D)$ functions and that

$$
\begin{gathered}
\left\|J_{D^{\prime} D}(z) F \otimes G\right\|_{C_{0}(D)} \leqq m_{D}(z)\|F \otimes G\|_{L^{2}(D)} \\
m_{D}(z)=\sup _{p, D^{\prime}} m_{D^{\prime}}\left(z-p^{2}\right)\left[z+E_{D^{\prime}}-p^{2}\right]^{-1}\left\|V_{D^{\prime} D}\right\|
\end{gathered}
$$

$J_{D^{\prime} D}$ can be extended to all of $L^{2}(D)$.

Now we must prove that $\tilde{I}_{D}(-E)$ has an analytic continuation to a family of compact operators in some complex tubular domain.

Definition 12. If $D$ is a cluster decomposition of $\{12 \ldots N\}$ which has $k$ internal coordinates then $T_{D}(z) \subset C^{3 k}$ is the tube

$$
\left\{p+i q: \operatorname{Re} z-E_{D^{\prime}}>q_{D D^{\prime}}^{2} \text { for all decompositions } D^{\prime} \underset{\ddagger}{\supset} D\right\} .
$$

$-E_{D}=\inf \sigma\left(\hat{H}_{D}\right)$ and $q_{D^{\prime} D}^{2}$ is the length of the vector $\boldsymbol{q}_{D^{\prime} D}$ described in the appendix.

Note. When $\operatorname{Re} z^{\prime}>\operatorname{Re} z, T_{D}\left(z^{\prime}\right) \supset T_{D}(z)$.

Theorem 10. If every $V_{i j} \in L^{2}\left(R^{3}\right), \tilde{I}_{D}(-E)$ has an extension to an analytic family of $H S$ operators $\tilde{I}_{D}\left(z_{D},-E\right)$ when $z_{D} \in T_{D}(E)$. Each $\tilde{I}_{D}\left(z_{D},-E\right)$ maps $L^{2}(D)$ into $C_{0}(D)$.

Proof. Our induction hypothesis is:

For each $D^{\prime} \supsetneq D, \tilde{I}_{D^{\prime}}(z)$ has the properties

(1) It is an analytic $H S$ valued function of $z$.

(2) It has an analytic extension to $T_{D^{\prime}}(z)$.

(3) It maps $L^{2}\left(D^{\prime}\right)$ into $C_{0}\left(D^{\prime}\right)$.

$g_{D^{\prime}}(z)$ is an analytic family of bounded operators on $L^{2}\left(D^{\prime}\right)$ and has property (2). We also assume

(4) When $z$ is more than an arbitrary minimum distance from $\sigma_{c}\left(\hat{H}_{D^{\prime}}\right)$, $\left\|\tilde{I}_{D^{\prime}}(z)\right\|_{H S}$ is uniformly bounded.

(5) $\left[\tilde{I}_{D^{\prime}}(z) f\right]\left(p_{D^{\prime}}\right)$ is a continuous function of both $z$ and $p_{D^{\prime}}$.

(6) Both $\tilde{I}_{D^{\prime}}(z)$ and $g_{D^{\prime}}(z)$ have the translation property. 
We can start the induction with $D=\{(12),(3), \ldots,(N)\}$. This is just the two body situation and has already been checked. Recall Eq. (26). We extend $\tilde{J}_{D^{\prime} D}(-E)$ to $\tilde{J}_{D^{\prime} D}\left(z_{D},-E\right)$ by defining

$$
\begin{aligned}
{\left[\tilde{J}_{D^{\prime} D}\left(z_{D},-E\right) a \otimes b\right]\left(p_{D^{\prime}}, p\right) } & \\
& =\left[\tilde{I}_{D^{\prime}} g_{D^{\prime}}\left(z_{D^{\prime}},-E-(p+z)^{2}\right) a\right]\left(p_{D^{\prime}}\right) \cdot\left[V_{D^{\prime} D} b\right](p)
\end{aligned}
$$

where

$$
\begin{gathered}
a \in L^{2}\left(D^{\prime}\right), \quad b \in L^{2}\left(R^{3}\right) . \\
p_{D}=\left(p_{D^{\prime}}, p\right) \quad \text { and } \quad z_{D}=\left(z_{D^{\prime}}, z\right) .
\end{gathered}
$$

If $z_{D} \in T_{D}(E)$ then $z_{D^{\prime}} \in T_{D^{\prime}}(E)$. Again $\tilde{J}_{D^{\prime} D}\left(z_{D},-E\right)$ extends to a $H S$ operator whenever $z_{D} \in T_{D}(E)$. In (30), $V_{D^{\prime} D} b$ is already a $C_{0}$ function of $p$. By induction $\left[\tilde{I}_{D^{\prime}} g_{D^{\prime}}\left(-E-(z+p)^{2}\right) b\right]\left(p_{D^{\prime}}\right)$ is a $C_{0}$ function of $p$ and $p_{D^{\prime}}$. Properties 4,5 and 6 are easily checked. So $\tilde{I}_{D}(-E)$ has all the right properties. It is a little more complicated to prove these for $g_{D}$. Equation (24) becomes

$$
g_{D}(-E)=\Delta_{D}(-E)+\tilde{I}_{D}(-E) g_{D}(-E)
$$

where $\Delta_{D}(-E)$ is $\sum_{D^{\prime} \supset D} \Delta_{D^{\prime} D}(-E) \Delta_{D^{\prime} D}(-E)$ is the extension to $L^{2}(D)$ of the operator defined by

$$
\left[\Delta_{D^{\prime} D}(-E) a \otimes b\right]\left(p_{D}\right)=\left[\tilde{I}_{D^{\prime}} g_{D^{\prime}}\left(-E-p^{2}\right) a\right]\left(p_{D^{\prime}}\right) \cdot b(p)
$$

where $a \in L^{2}\left(D^{\prime}\right), b \in L^{2}\left(R^{3}\right)$ and $p_{D}=\left(p_{D^{\prime}}, p\right)$. Each $\Delta_{D^{\prime} D}(-E)$ can be extended to an analytic family of bounded operators $\Delta_{D^{\prime} D}\left(z_{D},-E\right)$ on $L^{2}(D)$. Each $\tilde{I}_{D}$ can be extended to $\tilde{I}_{D}\left(z_{D},-E\right)$. We might hope to define an extension of $g_{D}$ by

$$
g_{D}\left(z_{D},-E\right)=\left[1-\tilde{I}_{D}\left(z_{D},-E\right)\right]^{-1} \cdot \Delta_{D}\left(z_{D},-E\right) .
$$

This will only be possible when $1 \notin \sigma\left[\tilde{I}_{D}\left(z_{D},-E\right)\right]$. Since $\tilde{I}_{D}$ is built from lower order kernels it has the translation property. This combined with the analytic Fredholm theorem shows that $1 \in \sigma\left[\tilde{I}_{D}\left(z_{D},-E\right)\right]$ if and only if $1 \in \sigma\left[\tilde{I}_{D}(-E)\right]$. In this case $-E$ is called an exceptional point of $\tilde{I}_{D}$. The exceptional points are isolated. If $-E$ is not exceptional (32) is an extension of $g_{D}$ with property (2). If $-E$ is an exceptional point there is an apparent singularity in (32). This is however a removable singularity. There is a neighbourhood $N$ which contains no other exceptional points besides $-E$. When $z \in N-\{-E\}$ we can define $g_{D}(\omega, z)$ for $\omega \in T_{D}(z)$. We also know that the equation

$$
g_{D}(x,-E)=\Delta_{D}(x,-E)+\tilde{I}_{D}(x,-E) g_{D}(x,-E)
$$


has a solution when $x$ is real, viz.,

$$
g_{D}(x,-E)=U_{D}(x) g_{D}(-E) U_{D}(-x)
$$

since $-E \notin \sigma\left(\hat{H}_{D}\right), T=\bigcap_{z \in N-\{-E\}} T_{D}(z)$ can be made arbitrarily close to $T_{D}(E)$ by taking $N$ sufficiently small.

This situation is an example of Hartogs basic phenomenon. We illustrate this with scalar valued analytic functions. Everything extends to operator valued functions.

We have a function $F(z, w)$ of two complex variables which

(1) is analytic in both $z$ and $w$ when $z \in T$ and $w \in N-\{0\}$

(2) when $z$ is real is analytic in $w$ around $w=0$.

(Here $T$ and $N$ are open neighbourhoods of 0 in the appropriate complex spaces.)

Then any singularity in $F(z, w)$ at $w=0$ is removable.

Proof. Bochner and Martin [2, p. 141].

Now we have only to prove the translation property for $g_{D}$.

$$
\begin{aligned}
U_{D}(t) g_{D}(z,-E) U_{D}(t)^{-1}= & \Delta_{D}(z+t,-E) \\
& +\tilde{I}_{D}(z+t,-E) U_{D}(t) g_{D}(z,-E) U_{D}(t)^{-1}
\end{aligned}
$$

On solution of (34) is $g_{D}(z+t,-E)$. If $-E$ is not an exceptional point the solution of (34) is unique, and so

$$
U_{D}(t) g_{D}(z,-E) U_{D}(t)^{-1}=g_{D}(z+t,-E)
$$

$g_{D}(z,-E)$ is analytic in both $z$ and $E$ and the exceptional points are isolated so (35) will hold everywhere. This completes the proof.

One more lemma is needed.

Lemma 10. (a) If all the interparticle potentials $V_{i j} \in L^{2}\left(R^{3}\right)$ and if $f \in L^{2}(D)$ then for each fixed $p$

$$
\left[\tilde{I}_{D}(z,-E) f\right](p)
$$

is an analytic function of $z$ in $T_{D}(E)$.

(b) If $f_{z}$ is an $L^{2}(D)$ valued analytic function when $z \in T_{D}(E)$ then $\left[\tilde{I}_{D}\left(z_{D},-E\right) f_{z}\right](p)$ is an analytic function of $z$ for fixed $p$.

Proof. (a) follows by examining each individual term $J_{D^{\prime} D}$ on the appropriate functions in $L^{2}(D)$. (b) is a simple extension of (a).

Finally we restate these results when $D=(12 \ldots N)$. This is the really important case. 
Corollary to Theorem 10. When the interparticle potentials $V_{i j} \in L^{2}\left(R^{3}\right)$ and then

(a) $I(-E)$ has an analytic extension to a family of $H S$ operators $I(z,-E)$ for $z \in T(E)$.

(b) $I(z,-E)$ maps $L^{2}\left(R^{3 N-3}\right)$ into $C_{0}\left(R^{3 N-3}\right)$.

(c) If $f_{z}$ is an analytic family of functions in $L^{2}\left(R^{3 N-3}\right)$ then $\left[I(z,-E) f_{2}\right](p)$ is an analytic function of $z$ for each fixed $p \in R^{3 N-3}$.

Proof. A restatement of Theorem 10 and Lemma 10.

\section{Section III. Decay in Several Particle Systems}

The method of proof for several particle systems is exactly the same as that for two particle systems. We have almost finished the proof by proving Theorem 10. One geometric lemma is needed.

Lemma 11. The tubular domain

$$
\begin{aligned}
S(E)= & \left\{p+i q \in C^{3 N-3}:|q|^{2}<E-E_{0},\right. \\
& \left.-E_{0}=\inf \sigma_{c}(\hat{H})\right\}
\end{aligned}
$$

lies inside $T(E)$.

Proof. If $p+i q \in T(E)$ then for each cluster decomposition $D^{\prime}$

$$
\left|q_{D^{\prime}}\right|^{2}<E-E_{D^{\prime}}, \quad-E_{D^{\prime}}=\inf \sigma\left(\hat{H}_{D^{\prime}}\right) .
$$

Hunziker's theorem says that $-E_{0}<-E_{D^{\prime}}$. So if $|q|^{2}<E-E_{0}$, (36) is always true.

Theorem 11. If $\hat{H}=\hat{H}_{0}+\sum_{i<j} V_{i j}$ is the Hamiltonian for a system of $N$ particles interacting through $L^{2}$ potentials and if

then

$$
\hat{H} \psi=-E \psi, \quad-E<-E_{0}=\inf \sigma_{c}(\hat{H})
$$

$$
\psi \in D\left[e^{\theta \sqrt{2 M\left(E-E_{0}\right)} r(x)}\right]
$$

for each $\theta: 0 \leqq \theta<1 . M$ is the total mass of the system and $r(x)$ is defined by $\mathrm{Mr}^{2}(x)$ being the moment of inertia of the system of $N$ particles when their relative configuration is specified by the vector $x \in R^{3 N-3}$.

Proof. $\psi$ satisfies the Weinberg-van Winter equation

$$
\psi=I(-E) \psi .
$$

$I(-E)$ has an extension to an analytic family of compact operators in $S(E)$ and since $1 \in \sigma[I(0,-E)], 1 \in \sigma[I(z,-E)]$ for every $z \in S(E)$. The analytic family of projections $P(z)$ onto these eigenspaces have the 
translation property and so Theorem 2 gives an extension of $\hat{\psi}$ to an analytic family of $L^{2}$ functions $\hat{\psi}_{z}$. Theorem 10 enables us to conclude that each $\hat{\psi}_{z}$ is a $C_{0}$ function and that $\hat{\Psi}(z)=\hat{\psi}_{z}(0)$ is an analytic extension of $\hat{\psi}(p)$ to $S(E)$. The slices of $\hat{\Psi}$ parallel to $R^{3 N-3}$ are square integrable and so we can apply Paley and Wiener's theorem. To complete the proof we need only reinsert the mass factors that have been ignored so far.

Suppose $p_{1} \ldots p_{N-1}$ is a set of Jacobi coordinates for the momenta of the $N$ particles in their centre of momentum frame and that

If

$$
\hat{H}_{0}=\sum_{i=1}^{N-1} \frac{1}{2} A_{i} p_{i}^{2} .
$$

$$
\varrho_{i}=\left(\frac{1}{2} A_{i}\right)^{\frac{1}{2}} p_{i} \quad \text { then } \quad \hat{H}_{0}=\sum_{i=1}^{N-1} \varrho_{i}^{2} .
$$

If $x_{1} \ldots x_{N-1}$ is a set of conjugate position coordinates to $p_{1} \ldots p_{N-1}$ we can define a set of transformed $x^{\prime} s$ by

$$
X_{i}=\left(\frac{1}{2} A_{i}\right)^{-\frac{1}{2}} x_{i} .
$$

Then $\boldsymbol{p} \cdot \boldsymbol{x}=\boldsymbol{\varrho} \cdot \boldsymbol{X}$. So far we have dealt with the coordinates $\left(\varrho_{i}: i=1 \ldots N-1\right)$ and we have shown that if $\theta$ if defined by

$$
\hat{\theta}\left(\varrho_{1} \ldots \varrho_{N-1}\right)=\hat{\psi}\left(p_{1} \ldots p_{N-1}\right)
$$

then $\theta(X)$ decays as $e^{-\sqrt{E-E_{0}}|X|}$.

Now

$$
\begin{aligned}
\theta(X) & =\int e^{i \varrho \cdot x} \hat{\theta}(\varrho) d \varrho \\
& =\int e^{i p \cdot x} \hat{\psi}(p) d p=\psi(x) .
\end{aligned}
$$

So

$$
\psi(x) \in D\left[e^{\theta \sqrt{E-E_{0}}|X|}\right]
$$

for each $\theta: 0 \leqq \theta<1$. We now have to calculate $|X|$ in terms of the original coordinates $x_{j}$ and the masses $m_{j}$

$$
|X|^{2}=2 \sum_{j=1}^{N-1} A_{j}^{-1} x_{j}^{2}=2 Q(x) .
$$

$Q(x)$ is the moment of inertia of the $N$ particles about their centre of mass. We can prove this by induction. $p_{N-1}$ is the relative momentum of two clusters $C_{1}$ and $C_{2}$. The remaining momenta are internal momenta of these two clusters. $x_{N-1}$ can be interpreted in the same way. Then

$$
Q(x)=Q_{C_{1}}(x)+Q_{C_{2}}(x)+A_{N-1}^{-1} x_{N-1}^{2} .
$$


If all the particles in $C_{1}$ have the same velocity and those in $C_{2}$ the same velocity then only $p_{N-1}$ is nonzero. This shows that

$$
A_{N-1}=M_{C_{1}}^{-1}+M_{C_{2}}^{-1} \text {. }
$$

Using (40) and (41) we complete the proof.

When the potentials are in $L^{2}+L_{\varepsilon}^{\infty}$ we again choose potentials $V_{i j}^{(n)}$ in $L^{2}$ which converge in $L^{\infty}$ to $V_{i j}$. The corresponding kernels $I_{n}(z,-E)$ converge to $I(z,-E)$ uniformly on compact subsets of $T(E)$. This is enough to extend the two body arguments to several particle systems. For Rollnik potentials we extend the symmetrized Weinberg-van Winter equations (19), (20). Again the Rollnik potentials $V_{i j}$ are approximated in $R$ norm by potentials in $R \cap L^{2}$. The symmetrized kernel $I_{s}\left(V_{i j}\right)$ depends continuously on the potentials $V_{i j}$ since

$$
\left\|I_{s}\left(V_{i j}\right)-I_{s}\left(W_{i j}\right)\right\| \leqq C_{N} \sup _{i, j}\left\|V_{i j}-W_{i j}\right\|_{R} .
$$

With these remarks every two body argument generalises and we are lead to the final theorem.

Theorem 12. $\hat{H}=\hat{H}_{0}+\sum_{i<j} V_{i j}$ is the Hamiltonian for a system of $N$ particles interacting through potentials in $R+L_{\varepsilon}^{\infty} \cdot \psi$ is an eigenfunction of $\hat{H}$ at energy $-E$. $-E$ is an isolated eigenvalue if $\hat{H}$.

Then

for each

$$
\psi \in D\left[e^{\theta \sqrt{2 M\left(E-E_{0}\right)} r(x)}\right]
$$

$$
\theta: 0 \leqq \theta<1, \quad-E_{0}=\inf \sigma_{c}(\hat{H}) .
$$

$M$ is the total mass of the system and $\mathrm{Mr}^{2}(x)$ is the moment of inertia of the $N$ particles about their centre of mass.

Remarks. (a) It is also possible to apply this method to spin dependent forces of the types considered by van Winter and Brascamp [22] or Balslev and Combes [24, 21].

(b) If the underlying space is $R^{n}, n>3$, the basic result is unchanged provided the potentials are in some $L^{p}$ space for $p>n / 2$. If $V L^{p}, p>n / 2$, $V$ is Kato tiny relative to $H_{0}$ [23] and is also $H_{0}$ compact. An approximation argument gives an extension to the spaces $L^{p}+L_{\varepsilon}^{\infty}$.

(c) Using results of Kato [8] on the boundedness and Holder continuity of wave functions for a wide class of potentials, Ahlrichs [1] proved a pointwise exponential bound. The rate of decay is not however the best possible. Recently Simon [25] has given a momentum space of Kato's result for a slightly different class of potentials. For this class he has strengthened the $L^{2}$ decay given here to pointwise decay with the same exponent. 
(d) Combes and Thomas [26] have recently given a more direct proof. It applies to dilatation analytic potentials. This method applies to point eigenvalues embedded in the continuum (but away from thresholds). This is an important advantage over the present method.

Acknowledgements. It is a pleasure to thank Professor B. Simon for suggesting this problem and to acknowledge my debt to him as a teacher and adviser. Part of the work contained here was done while I was at the Belfer Graduate School, Yeshiva University, and was supported by USAFOSR contract F 44620-71-C0013, and I thank Professor J. L. Lebowitz for his support during this period. I also thank Professors R. Ahlrichs, J. M. Combes and L. Thomas for allowing me to see their work before publication, and Professor L. O'Raifeartaigh for his hospitality at the Dublin Institute for Advanced Studies.

\section{Appendix. Kinematics of Several Particle Systems}

We have $N$ particles with momenta $p_{i}$ and masses $m_{i}(1 \leqq i \leqq N)$. Their kinetic energy is $H_{0}$ and $\hat{H}_{0}$ is their kinetic energy relative to their centre of mass. With any string of cluster decompositions we can associate a set of Jacobi coordinates which diagonalize $\hat{H}_{0}$.

Definition A1.S is a string of cluster decompositions $\left\{D_{N}, D_{N-1}, \ldots, D_{k}\right\}$. The clusters in $D_{j}$ are labelled $C_{j}(1), \ldots, C_{j}(j) . D_{j-1}$ is obtained from $D_{j}$ by combining the clusters $C_{j}[a(j)]$ and $C_{j}[b(j)]$ together to form a single cluster. Then the internal Jacobi coordinates for $S$ are

$p_{j-1}=$ relative momentum of $C_{j}[a(j)]$ and $C_{j}[b(j)]$ in $D$ for $N \geqq j \geqq k+1$,

$$
\text { i.e. }\left(M_{a}+M_{b}\right) p_{j-1}=M_{a} P_{b}-M_{b} P_{a} \text {. }
$$

$M_{a}, P_{a}$ and $M_{b}, P_{b}$ are the total mass and momentum of $C_{j}[a(j)]$ and $C_{j}[b(j)]$ respectively. To completely specify the $N-1$ relative momenta we supplement these internal coordinates by a further $k-1$ vectors. There are many ways of doing this. Viewing the $k$ clusters in $D$ as particles with specified masses and momenta we can take any string $S^{\prime}$ of cluster decompositions of $\{1 \ldots k\}$.

$$
\begin{aligned}
& S^{\prime}=\left\{D_{k}, \ldots, D_{i}\right\} \\
& D_{k}=\{(1),(2), \ldots,(k)\} \\
& D_{1}=\{(12 \ldots k)\} \\
& D_{j} \supset_{1} D_{j-1} .
\end{aligned}
$$

For any string $S^{\prime}$ we can take the set of relative Jacobi coordinates $\left\{q_{1} \ldots q_{k-1}\right\}$. The total set $\{\boldsymbol{q}, \boldsymbol{\varrho}\}=\left\{q_{1}, \ldots, q_{k-1} ; \boldsymbol{\varrho}_{k}, \ldots, \boldsymbol{\varrho}_{N-1}\right\}$ of momenta form a set of Jacobi coordinates for the system.

Note. The particular value of $\boldsymbol{q}$ depends on the $S^{\prime}$ we choose. 
Theorem A1. For any set of Jacobi coordinates we can find positive numbers $A_{i}$ so that

$$
\hat{H}_{0}=\sum_{i=1}^{k-1} A_{i} q_{i}^{2}+\sum_{i=k}^{N-1} A_{i} \varrho_{i}^{2} .
$$

Proof. Induction.

Definition $A 2$. If a string $S$ ends with a decomposition $D$ we define vectors $\boldsymbol{p}_{\boldsymbol{D}}$ and $\boldsymbol{q}_{D}$ by

$$
\begin{aligned}
& \boldsymbol{p}_{D}=\left\{A_{k}^{\frac{1}{2}} \varrho_{k}, \ldots, A_{N-1}^{\frac{1}{2}} \varrho_{N-1}\right\}, \\
& \boldsymbol{q}_{D}=\left\{A_{1}^{\frac{1}{2}} q_{1}, \ldots, A_{k-1}^{\frac{1}{2}} q_{k-1}\right\} .
\end{aligned}
$$

Note. (a) $\hat{H}_{0}=p_{D}^{2}+q_{D}^{2}$.

$p_{D}^{2}$ is the internal kinetic energy of the clusters and $q_{D}^{2}$ is the relative kinetic energy of the clusters. Since $\boldsymbol{p}_{D}$ is fixed by $S, \boldsymbol{q}_{D}^{2}$ must be independent of the choice of $S^{\prime}$.

(b) If $D^{\prime} \supset D, D$ containing $l$ clusters and $D k$ clusters $(l>k)$, we can find a string $S=\left\{D_{N} \ldots D_{l} \ldots D_{k}\right\}$ where $D_{l}=D^{\prime}, D_{k}=D$. Then

and

$$
\begin{aligned}
\boldsymbol{p}_{D^{\prime}} & =\left\{A_{l}^{\frac{1}{2}} \varrho_{l}, \ldots, A_{N-1}^{\frac{1}{2}} \varrho_{N-1}\right\} \\
\boldsymbol{p}_{D} & =\left\{A_{k}^{\frac{1}{2}} \varrho_{k}, \ldots, A_{N-1}^{\frac{1}{2}} \varrho_{N-1}\right\} \\
\boldsymbol{p}_{D D^{\prime}} & =\left\{A_{k}^{\frac{1}{2}} \varrho_{k}, \ldots, A_{l+1}^{\frac{1}{2}} \varrho_{l+1}\right\} .
\end{aligned}
$$

Then (1) $\boldsymbol{p}_{D}^{2}=\boldsymbol{p}_{D^{\prime}}^{2}+\boldsymbol{p}_{D D^{\prime}}^{2}$ and so $\boldsymbol{p}_{D D^{\prime}}^{2}$ is independent of $S^{\prime}$. (2) If $D^{\prime} \supset D, p_{D}^{2} \geqq \varrho_{D^{\prime}}^{2}$.

\section{References}

1. Ahlrichs, R.: Preprint 1972, Institut für Physikalische Chemie der Universität Karlsruhe

2. Bochner, S., Martin,W.T.: Several complex variables. Princeton: University Press 1948

3. Combes, J. M.: Nuovo Cimento 64 A, 111 (1969)

4. Federbush, P. G.: Phys. Rev. 148, 1551 (1966)

5. Hepp, K.: Helv. Phys. Acta 42, 425 (1969)

6. Hunziker, W. Phys. Rev. 135, B 800 (1964)

7. Hunziker, W.: J. Math. Phys. 7, 300 (1966)

8. Kato, T.: Commun. Pure and Appl. Math. 10, 151 (1957)

9. Newton, R. G.: Phys. Rev. 153, 1502 (1967)

10. Paley, R.E. A.C., Wiener, N.: Fourier transforms in the complex plane. Amer. Math. Soc. Colloq. Publn. 19 (1934)

11. Reed, M., Simon, B.: Methods of Modern Mathematical Physics (Vol. I). New York: Academic Press 1972

12. Alfaro, V.de, Regge, T.: Potential scattering. Amsterdam: North Holland 1965

13. Schnoll,I.E.: Mathematicheskii Sbornik 42, 84, 3 (1951)

Glazman,I.M.: Direct methods in the qualitative spectral analysis of singular differential operators. Israeli Program for Scientific Translations, Jerusalem 1965 
14. Simon, B. : Quantum mechanics for hamiltonians defined as quadratic forms. Princeton University Press 1971

15. Slaggie,E. L., Wichmann,E.H.: J. Math. Phys. 3, 946 (1962)

16. Titchmarsh, E.C.: Introduction to the theory of the Fourier integral. Oxford: Oxford University Press 1937

17. van Winter, C.: Mat. Fys. Skr. Dan. Vid. Selsk. 1, 8 (1964); 1, 10 (1965)

18. Weinberg, S.: Phys. Rev. 133, 232 (1964)

19. Nelson, E.: Annal of Math. 70, 572 (1959)

20. Schminke, U.-W.: Math. Z. 11, 267 (1969)

21. Combes, J. M.: Commun. math. Phys. 12, 283 (1969)

22. van Winter, C., Brascamp, H.J.: Commun. math. Phys. 11, 19 (1968)

23. Nelson, E.: J. Math. Phys. 5, 332 (1964)

24. Balslev, E.: Math. Scand. 19, 193 (1966)

25. Simon, B.: IHES preprint, December 1972. Pointwise Bounds on Eigenfunctions and Wave Packets in $N$-Body Quantum Systems

26. Thomas, L.: Preprint, Université de Genève, Départment de Physique Théorique Combes, J. M.: Preprint, Department of Mathematics, Centre Universitaire de Toulon

A. J. O'Connor

School of Theoretical Physics

Dublin Institute for Advanced Studies

Dublin 4, Ireland 